\title{
Markers for distinguishing Orostachys species by SYBR Green-based real-time PCR and verification of their application in commercial $\boldsymbol{O}$. japonica food products
}

\author{
Jun An ${ }^{1} \cdot$ Jun-Cheol Moon ${ }^{2} \cdot$ Cheol Seong Jang ${ }^{1}$
}

Received: 23 April 2018/Accepted: 12 July 2018/Published online: 18 July 2018

(C) The Korean Society for Applied Biological Chemistry 2018

\begin{abstract}
Human consumption of plant functional foods has been rapidly increasing owing to the health benefits they provide. In particular, in Korea, the plant Orostachys japonica has attracted attention for its anticancer and other effects. Of the 12 established Orostachys species, only three (viz., $O$. iwarenge, $O$. malacophyllus, and $O$. japonica) have been allowed for use as foods in Korea. In this study, 12 species-specific primer sets based on single nucleotide polymorphisms of five chloroplast genes and one nuclear gene were developed to discriminate Orostachys species through quantitative real-time PCR (qPCR) analysis with SYBR Green staining. The efficiencies of the designed primer pairs in amplifying the target species ranged from 80 to $110 \%$, with strong correlation coefficients $\left(R^{2}>0.99\right)$, whereas no clear correlation coefficient was evident for the non-target species. In order to verify the specificity of the 12 developed Orostachys-specific primers, binary mixtures of the DNAs (tenfold serially diluted samples) from the target species and each of the other non-target species were generated for qPCR analysis, with results suggesting that the primers could clearly discriminate at least $0.1 \%$ of $O$. japonica DNA $(10 \mathrm{pg})$ in the mixtures. With regard to the feasibility of the developed
\end{abstract}

Electronic supplementary material The online version of this article (https://doi.org/10.1007/s13765-018-0383-3) contains supplementary material, which is available to authorized users.

Cheol Seong Jang

csjang@kangwon.ac.kr

1 Plant Genomics Laboratory, Department of Bio-resources Sciences, Kangwon National University, Chuncheon 24341, Republic of Korea

2 Agriculture and Life Sciences Research Institute, Kangwon National University, Chuncheon 24341, Republic of Korea
qPCR system for detecting Orostachys species in $O$. japonica food products, $O$. japonica DNA was detected in all eight commercial products tested, with low $\mathrm{Ct}$ values $(<20)$, whereas none of the other Orostachys species DNAs were detected, confirming that the tested foods contained only $O$. japonica. Therefore, developed primers and qPCR conditions would be useful for verifying the authenticity of commercial $O$. japonica food products.

Keywords Commercial O. japonica foods - Orostachys . Real-time PCR · Species-specific DNA markers

\section{Introduction}

Functional foods are defined as natural food products that contain bioactive compounds [1]. Recently, the human consumption of plant functional foods has been rapidly increasing owing to the health benefits that they provide [2]. For example, plant flavonoids have attracted attention for their cancer chemopreventive effects [3]. However, the plant functional food markets are faced with fraud and adulteration issues, such as the addition of morphologically similar low-cost raw materials in food processing, because it is difficult to discriminate between spurious and authentic raw materials [4]. For example, the root tissue of Cynanchum auriculatum has been known to be sold as that of the morphologically similar Cynanchum wilfordii. In Korea, about $60 \%$ of the C. wilfordii products on the market contain $C$. auriculatum, which has created a major problem in the Korean market [5]. Therefore, it is necessary to develop various technologies to protect consumers from food fraud such as mislabeling and unintended mixtures. 
Genus Orostachys comprises perennial crassulacean acid metabolism plants that grow in Korea, Japan, and Russia and adapt easily to drought and cold environments [6]. The genus contains more than 12 acknowledged species (http://www.theplantlist.org), among which $O$. japonica has been confirmed to have several efficacies, such as antipyretic, hemostatic, diuretic, apoptotic cell death, and anticancer effects [7-9]. As a result, various commercial food products containing $O$. japonica ingredients in raw, powder, pouch, and tablet forms have been developed and sold in Korean markets. Aside from $O$. japonica, $O$. iwarenge and O. malacophyllus are the only other Orostachys species that have been allowed for use as foods by the Ministry of Food and Drug Safety of Korea. However, Orostachys species that have been dried are difficult to distinguish by the naked eye. Therefore, it is necessary to develop methods to discriminate the species in order to prevent their indiscriminate ingestion in foods.

Most of the chloroplasts, which play an important role in photosynthesis, have a circular-structured genome of $120-170 \mathrm{~kb}$ in size $[10,11]$. Chloroplast genomes have been widely used in evolutionary studies and for plant species identification. For example, the maturase K (matK) gene is highly conserved among different plants and has been used for species identification, especially by the DNA barcoding method [12]. In addition, a host of studies have reported the determination of species-level relationships by using the mat $K$ gene [13-15]. Other chloroplast genes, such as $\operatorname{trn} T-L, \operatorname{trnS}-G, n d h F, \operatorname{rpoC} 2$, and $y c f 2$, have been used for species identification [16-18]. Alternatively, the internal transcribed spacer (ITS) region of the $18 \mathrm{~S}-5.8 \mathrm{~S}-26 \mathrm{~S}$ nuclear ribosomal cistron has also been widely used for species identification owing to its intragenomic uniformity, but inter-genomic variability [19].

DNA-based polymerase chain reaction (PCR) analyses have been widely used owing to their economical and timesaving advantages. In particular, the high specificity and sensitivity of the quantitative real-time PCR (qPCR) assay allow the detection of very low levels of target DNA sequences in commercial foods. Many research studies have used qPCR for the detection of ingredients in commercial foods, such as pistachio [20], C. wilfordii [5], and rice [21].

In this study, we developed species-specific molecular markers from chloroplast genes and the nuclear region for the discrimination of six Orostachys species and verified their application in commercial $O$. japonica-containing food products.

\section{Materials and methods}

\section{Samples}

A total of six species of Orostachys were used in this study (Table 1). All plants were obtained from three botanical gardens. All commercial food products were purchased from farming corporations (Table 2).

\section{Genomic DNA extraction}

Total genomic DNA was extracted from leaves of the Orostachys plants and from all commercial food products using the i-genomic Plant DNA Extraction Mini Kit (iNtRON Biotechnology, Seongnam, Korea) according to the manufacturer's protocol. The quantity of the extracted genomic DNA was measured using the Qubit 2.0 Fluorometer (Invitrogen ${ }^{\mathrm{TM}}$, Life Technologies, Grand Island,

Table 2 Commercial Orostachys food products used in this study

\begin{tabular}{lll}
\hline Samples & Sample types & Source \\
\hline 1 & Powder & Gongju, Chungnam, Korea \\
2 & Powder & Haenam, Jeonnam, Korea \\
3 & Dried & Haenam, Jeonnam, Korea \\
4 & Dried & Yeoju, Gyeonggi, Korea \\
5 & Dried & Iksan, Jeonbuk, Korea \\
6 & Dried & Yanggu, Gangwon, Korea \\
7 & Powder & Gimcheon, Gyeongbuk, Korea \\
8 & Dried & Jinju, Gyeongnam, Korea \\
\hline
\end{tabular}

Table 1 Orostachys plant species used in this study

\begin{tabular}{lll}
\hline Species & Edibility $^{\mathrm{a}}$ & Source \\
\hline O. japonica & Allowed & Kangwondo Wasong Farm, Yanggu, Gangwon, Korea \\
O. iwarenge & Allowed & Orostachys Garden, Gongju, Chungnam, Korea \\
O. malacophyllus & Allowed & Orostachys Garden, Gongju, Chungnam, Korea \\
O. latiellipticus & Not allowed & Orostachys Garden, Gongju, Chungnam, Korea \\
O. ramosus & Not allowed & Baekrimwo Garden, Daejeon, Chungnam, Korea \\
O. margaritifolius & Not allowed & Orostachys Garden, Gongju, Chungnam, Korea \\
\hline
\end{tabular}

${ }^{a}$ Based on the standards established by the Ministry of Food and Drug Safety of Korea 
NY, USA) with a Qubit dsDNA BR Assay Kit (Invitrogen, Life Technologies).

\section{Gene cloning and nucleotide sequence analysis}

The nucleotide sequences of six chloroplast genes and one nuclear region were used for the development of the species-specific markers. The Orostachys nuclear DNA sequences were downloaded from the National Center for Biotechnology Information (NCBI; https://www.ncbi.nlm. nih.gov/), whereas the chloroplast DNA sequences were obtained from the National Instrumentation Center for Environmental Management (NICEM, Korea; https:// nicem.snu.ac.kr). The sequences were aligned using ClustalW2 (ftp://ebi.ac.uk/pub/software/clustalw2/). BioEdit 7.2 software (Ibis Biosciences, Carlsbad, CA, USA) was employed for editing the sequence alignments. For cloning of the ITS region, primer pairs were designed using Beacon Designer $^{\text {TM }}$ (PRIMER Biosoft, Palo Alto, CA, USA) and were synthesized by a commercial service (Macrogen,
Seoul, Korea) (Table S1). The conventional PCR was performed using TaKaRa Ex $\mathrm{Taq}^{\mathrm{TM}}$ DNA polymerase (TaKaRa Bio Company, Kusatsu, Shiga, Japan), with the following conditions: $5 \mathrm{~min}$ at $95^{\circ} \mathrm{C}$, followed by 35 cycles of $10 \mathrm{~s}$ at $95^{\circ} \mathrm{C}, 30 \mathrm{~s}$ at $59{ }^{\circ} \mathrm{C}$, and $1 \mathrm{~min}$ at $72{ }^{\circ} \mathrm{C}$, and finally, $5 \mathrm{~min}$ at $72{ }^{\circ} \mathrm{C}$. The amplicons were cloned into the RBC T\&A Cloning Vector (Real Biotech Corporation, Taipei, Taiwan) using the TaKaRa ligation mix (TaKaRa) according to the manufacturer's protocol. Plasmid DNA was purified using the Plasmid Mini-Prep Kit (Elpis Biotech, Daejeon, Korea), and the nucleotide sequence was analyzed by a commercial service (Macrogen) (Fig. 1).

\section{Quantitative real-time PCR analysis}

All primer pairs were designed using Beacon Designer and synthesized by a commercial service (Macrogen). The qPCR was performed in a final volume of $20 \mu \mathrm{L}$ using the QuantStudio 3 Real-Time PCR System (Applied

(A)

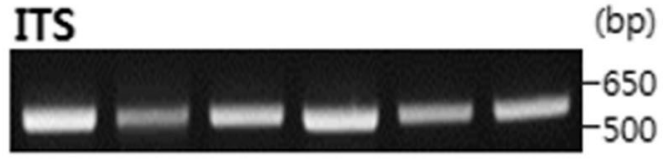

\section{$\begin{array}{llllll}1 & 2 & 3 & 4 & 5 & 6\end{array}$}

(B) ITS O.margaritifolius ITS O. japonica ITS O.latiellipticus ITSO.iwarenge Clustal Consensus
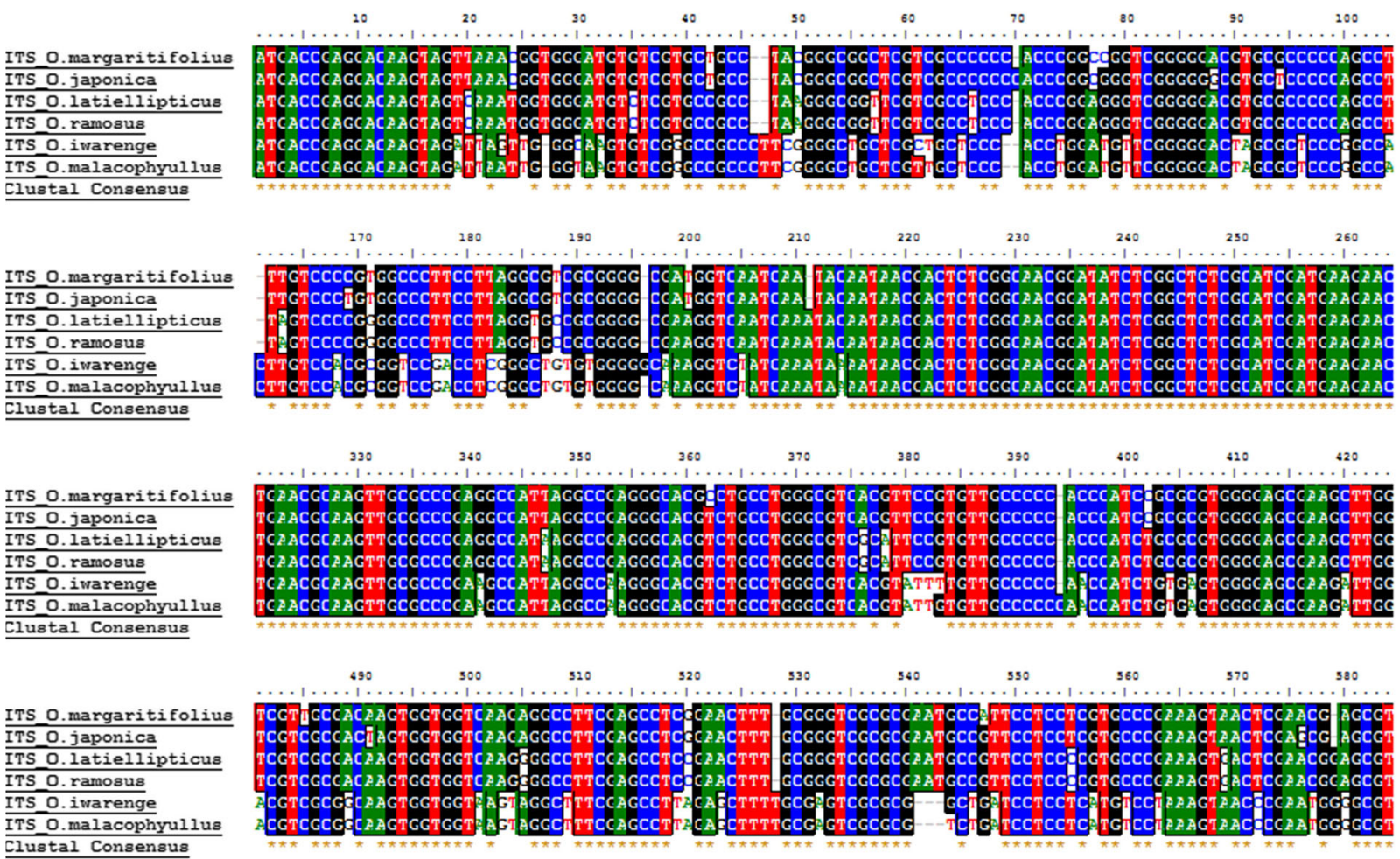

Fig. 1 (A) PCR products of the internal transcribed spacer (ITS) region of Orostachys species. 1: O. japonica; 2. O. iwarenge; 3. O. malacophyllus; 4. O. latiellipticus; 5. O. margaritifolius; 6. O. ramosus. (B) Sequence alignment of the ITS region from the Orostachys species 
Biosystems, Foster City, CA, USA) with SYBR Green dye staining. The reaction mixture contained $10 \mu \mathrm{L}$ of AccuPower ${ }^{\circledR} 2 \times$ GreenStar $^{\mathrm{TM}}$ qPCR Master Mix (Bioneer, Daejeon, Korea), 10 pmol of each primer set, and $10 \mathrm{ng}$ of genomic DNA, adjusted to a final volume of 20 $\mu \mathrm{L}$ with PCR-grade water. The qPCR conditions were as follows: $10 \mathrm{~min}$ at $95{ }^{\circ} \mathrm{C}$, followed by 40 cycles of $10 \mathrm{~s}$ at $95^{\circ} \mathrm{C}$, annealing time at the appropriate annealing temperature $\left(\mathrm{T}_{\mathrm{m}}\right)$ of each primer pair, and $30 \mathrm{~s}$ at $72{ }^{\circ} \mathrm{C}$. The PCR products were denatured at $95{ }^{\circ} \mathrm{C}$ for $15 \mathrm{~s}$ and then annealed at $60{ }^{\circ} \mathrm{C}$ for $1 \mathrm{~min}$. This step was followed by melt-curve analysis at temperatures ranging from 60 to $95{ }^{\circ} \mathrm{C}$, with increments of $3{ }^{\circ} \mathrm{C}$ every $30 \mathrm{~s}$. For sensitivity analysis, the DNA of each Orostachys species was diluted tenfold into five series (ranging from 0.001 to $10 \mathrm{ng} / \mu \mathrm{L}$ ) and subjected to qPCR. For the commercial $O$. japonica food products, each extracted DNA sample was diluted tenfold into three series (ranging from 0.1 to $10 \mathrm{ng} / \mu \mathrm{L}$ ) and subjected to qPCR.

\section{Determination of amplification efficiency, correlation coefficient, and limit of detection (LOD)}

To evaluate the correlation between cycle threshold $(\mathrm{Ct})$ and DNA concentration, standard curves were obtained using tenfold serially diluted DNA samples of the Orostachys species at concentrations of 0.001-10 ng. The correlation coefficient $\left(\mathrm{R}^{2}\right)$ was determined by using the linear regression method $\left(R^{2} \geq 0.98\right)$ [22]. The amplification efficiency was calculated on the basis of the standard curve using the equations $E=10^{-1 / \text { slope }}$, and efficiency $(\%)=(E-1) \times 100$. The limit of detection (LOD) was regarded as the analytical concentration at which the method detected the presence of a target nucleic acid in at least $95 \%$ of true-positive biological samples $(<5 \%$ of false-negative results) [23]. In order to confirm whether the developed methods work well in other laboratories, we performed an inter-laboratory validation in two other laboratories. The inter-laboratory validation in both laboratories was performed with the same PCR conditions, using the Rotor-Gene Q real-time PCR instrument (Qiagen, Hilden, Germany).

To evaluate the specificity of the $O$. japonica-specific primer set, we used binary mixtures of DNAs from $O$. japonica and other Orostachys species, and $O$. japonica powder and wheat flour $(\mathrm{mg} / \mathrm{g})$. The binary DNA mixtures of $O$. japonica and other Orostachys species were generated from tenfold serially diluted mixed DNA (10-0.01\%) samples. The $10 \mathrm{~g}$ plant/flour mixtures were prepared artificially by mixing $0.1-90 \%(10,100,1000$, and $9000 \mathrm{mg} / \mathrm{g}$ ) concentrations of $O$. japonica powder and wheat flour. The extracted DNA mixtures were serially diluted tenfold (0.01-10\%) with the initial DNA concentration $(10 \mathrm{ng})$ of each species.

(A)

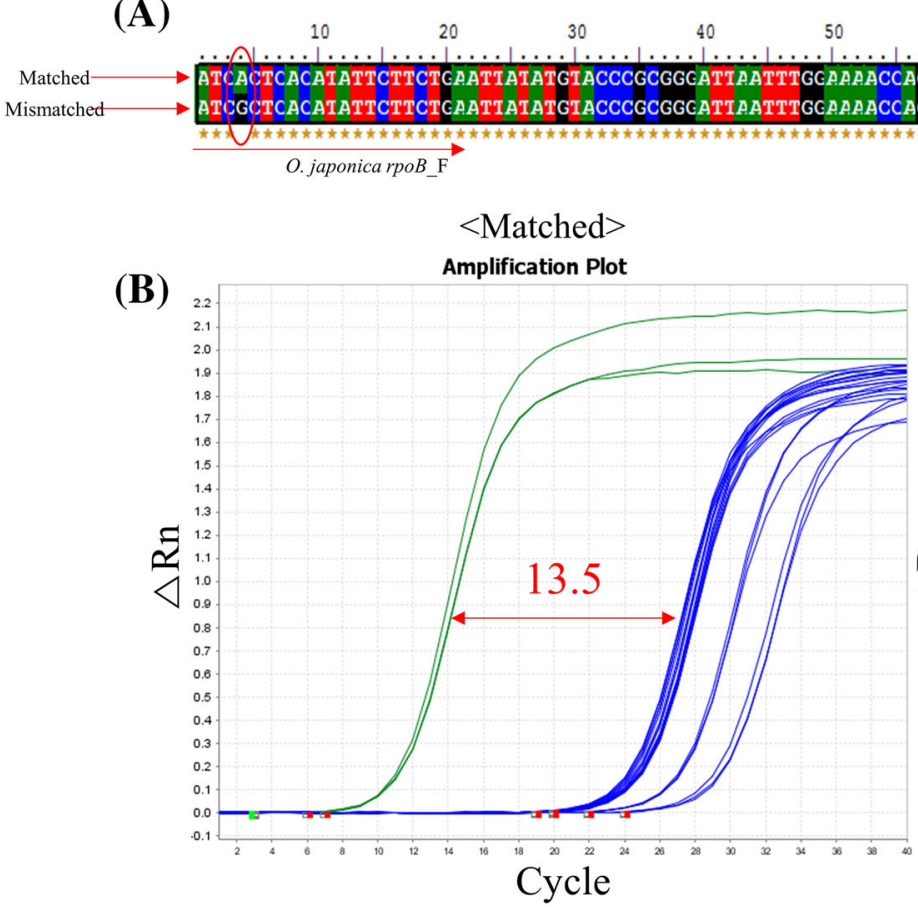

60

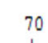

80

90

100

110

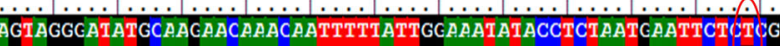
( O. japonicarpoB_R

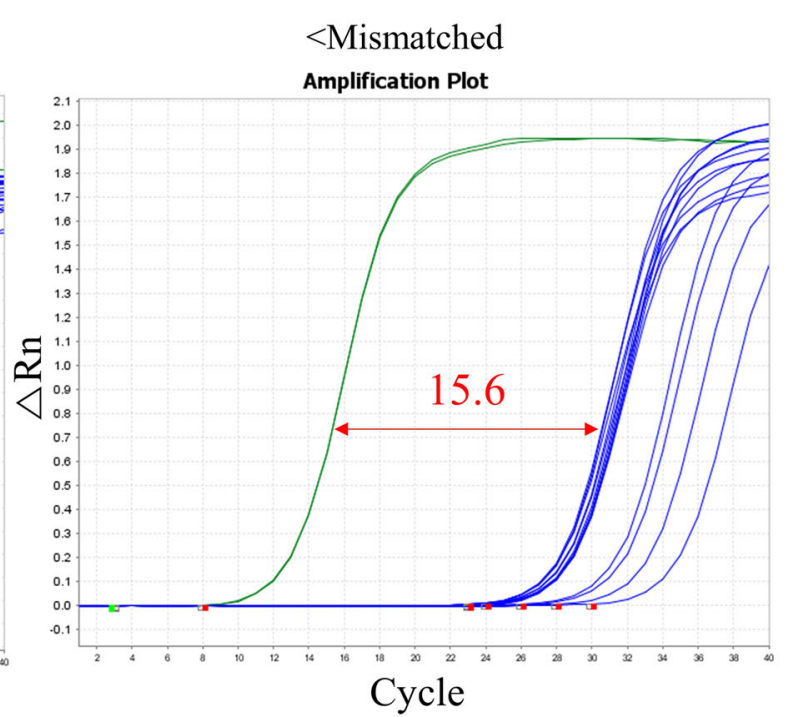

Fig. 2 (A) Mismatched sequence of $O$. japonica rpoB primer. (B) Comparison of Ct values for the amplification of target and non-target species before and after the addition of $O$. japonica mismatched $r p o B$ primer pairs. For all species, $10 \mathrm{ng}$ of DNA samples were used in the experiments 
Table 3 Target genes, primer sequences, amplicon sizes, and $T_{\mathrm{m}}$ values for the Orostachys species-specific qPCR developed in this study

\begin{tabular}{|c|c|c|c|c|c|c|}
\hline Target species & $\begin{array}{l}\text { Target } \\
\text { gene/region }\end{array}$ & Primer & Length (bp) & Sequence $\left(5^{\prime} \rightarrow 3^{\prime}\right)$ & Size (bp) & $T_{\mathrm{m}}\left({ }^{\circ} \mathrm{C}\right)$ \\
\hline \multirow{2}{*}{$\begin{array}{l}\text { Plant system } \\
\text { (positive control) }\end{array}$} & \multirow[t]{2}{*}{ 18S rRNA } & 18S rRNA_F & 25 & TCTGCCCTATCAACTTTCGATGGTA & \multirow[t]{2}{*}{137} & \multirow[t]{2}{*}{58} \\
\hline & & 18S rRNA_R & 25 & AATTTGCGCGCCTGCTGCCTTCCTT & & \\
\hline \multirow[t]{6}{*}{ O. japonica } & \multirow[t]{2}{*}{$\operatorname{trn} T-L$} & $\operatorname{trn} T-L \_\mathrm{F}$ & 24 & GAAACTACAGAAAGAAAGGATGAA & \multirow[t]{2}{*}{140} & \multirow[t]{2}{*}{61} \\
\hline & & $\operatorname{trn} T-L \_\mathrm{R}$ & 18 & CTCGGAATCGCTTCCTAC & & \\
\hline & \multirow[t]{2}{*}{$n d h F$} & $n d h F_{-} \mathrm{F}$ & 20 & CATCTATAAATCTTTTACAG & \multirow[t]{2}{*}{149} & \multirow[t]{2}{*}{51} \\
\hline & & $n d h F_{-} \mathrm{R}$ & 20 & TTTTTGAAAGATGAATAAAC & & \\
\hline & \multirow[t]{2}{*}{ rров } & $r p o B \_F$ & 21 & ATCGCTCACATATTCTTCTGA & \multirow[t]{2}{*}{116} & \multirow[t]{2}{*}{58} \\
\hline & & rpoB_R & 20 & CGEGGAGAATTCATTAGAGGT & & \\
\hline \multirow{8}{*}{$\begin{array}{l}\text { O. iwarenge and } O \text {. } \\
\text { malacophyllus }\end{array}$} & \multirow[t]{2}{*}{$m a t K$} & matK_F & 19 & CGAATCCATACTCGGTTTT & \multirow[t]{2}{*}{129} & \multirow[t]{2}{*}{58} \\
\hline & & matK_R & 20 & TGAATAGAAAAGCCTTCTAG & & \\
\hline & \multirow[t]{2}{*}{$\operatorname{trn} S-G$} & $\operatorname{trn} S-G \_\mathrm{F}$ & 20 & GTGATTTTTATCCAAATTTG & \multirow[t]{2}{*}{155} & \multirow[t]{2}{*}{55} \\
\hline & & $\operatorname{trnS}$ - $G \_\mathrm{R}$ & 21 & GATTTAGTTACGATTAGAAAG & & \\
\hline & \multirow[t]{2}{*}{$\operatorname{trn} Q-r p s 16$} & trnQ-rps16_F & 18 & TCACCTAGTGCATCTCGG & \multirow[t]{2}{*}{102} & \multirow[t]{2}{*}{62} \\
\hline & & trnQ-rps16_R & 23 & GGTTTCATAGAACAGATCAAGGT & & \\
\hline & \multirow[t]{2}{*}{$y c f 2$} & $y c f 2 \_\mathrm{F}$ & 18 & TGTGGGGCTAATAGTTTG & \multirow[t]{2}{*}{181} & \multirow[t]{2}{*}{58} \\
\hline & & $y c f 2 \_\mathrm{R}$ & 19 & TAACCAGGAACTTGTTCAG & & \\
\hline \multirow{8}{*}{$\begin{array}{l}\text { O. latiellipticus and } \\
\text { O. ramosus }\end{array}$} & \multirow[t]{2}{*}{$m a t K$} & matK_F & 19 & AGGTACGCCTCTCCTGATA & \multirow[t]{2}{*}{186} & \multirow[t]{2}{*}{62} \\
\hline & & matK_R & 23 & TCTAATAGTTGACTCCGTACAAA & & \\
\hline & \multirow[t]{2}{*}{$\operatorname{trn} T-L$} & $\operatorname{trn} T-L \_\mathrm{F}$ & 22 & GCATGTTATGTTTCTCATTCAC & 156 & 60 \\
\hline & & $\operatorname{trn} T-L \_\mathrm{R}$ & 20 & ACTTGAGGCTATGTCAATTC & & \\
\hline & $\operatorname{trnQ-rps16}$ & trnQ-rps16_F & 19 & ATTTGGGATTTAAATAGGG & 115 & 54 \\
\hline & & trnQ-rps16_R & 20 & AGTACTCCTTCTATAGTTAG & & \\
\hline & ITS & ITS_F & 18 & GAAGCGAAAATCGGACAT & 152 & 59 \\
\hline & & ITS_R & 19 & GCCEATTCACACCAAGTATC & & \\
\hline O. margaritifolius & $n d h F$ & $n d h F_{-} F$ & 18 & АААТTCСТCTGAАACTTG & 218 & 53 \\
\hline & & $n d h F_{-} \mathrm{R}$ & 18 & TTATAGTACTTCCCCTAG & & \\
\hline
\end{tabular}

Nucleotides in underlining letters indicate mismatched sequences in the designed primers

\section{Results and discussion}

\section{Orostachys nucleotide sequences analysis}

The sequences of seven chloroplast genes (viz., matK, $n d h F, r p o B, \operatorname{trnS}-G, \operatorname{trn} T-L$, trnQ-rps 16 , and ycf2) were obtained from NICEM (for six Orostachys chloroplast genome sequences, unpublished data). The ITS sequences of $O$. japonica were obtained from the NCBI. In order to amplify the ITS sequences of the six species, primers were designed with an appropriate $\mathrm{Tm}$ value $\left(59^{\circ} \mathrm{C}\right)$ (Table S1). The primers successfully amplified PCR products from all six species (Fig. 1A). The amplicons of the chloroplast genes and ITS regions were subsequently cloned and sequenced, and the nucleotide sequences were aligned using ClustalW2 in order to identify single nucleotide polymorphisms (SNPs) for comparison of differences among the six Orostachys species. Species-specific SNPs were found to distinguish each species in each set of alignments (e.g., for the ITS alignment, see Fig. 1B). However, no distinguishing SNPs were found between the sequences of the species pairs $O$. iwarenge and $O$. malacophyllus, and $O$. latiellipticus and $O$. ramosus. The species within these two species pairs might have diverged only recently, resulting in the lack of SNP differences between them. Previously, Kim and Park [24] reported that $O$. malacophyllus and $O$. iwarenge belonged to the same group on the basis of isoenzyme analysis. We therefore decided to develop species-specific primers for the $O$. iwarenge and $O$. malacophyllus, and $O$. latiellipticus and O. ramosus pairs, respectively.

\section{Real-time PCR primers design}

To develop species-specific primers, we retrieved speciesspecific SNPs from among the Orostachys species and used those as the basis for the primer design by the commercial program. However, because the nucleotide sequences of 
the Orostachys species are very similar, it was not easy to retrieve appropriate SNPs for $\mathrm{qPCR}$ primer design. It is very difficult to design primer pairs for the development of PCR-based molecular markers if the SNP has only one of the forward or reverse primers. Some researchers have suggested that these difficulties could be resolved by increasing the primer specificity, by adding mismatches to the base pairs around the SNPs [25, 26]. Therefore, we attempted such addition of mismatches to the base pairs of the designed primers for increasing the specificity. Consequently, 6 out of 12 primer pairs were confirmed as being a vast improvement over the originally designed primer pairs. For example, the mismatched $r p o B$ primer pair showed improvement of specificity (at least 15.6 cycles were rapidly amplified for $O$. japonica DNA as compared to the other species) over that of the original primers (at least 13.5 cycles) (Fig. 2). The designed and modified primer pairs are listed in Table 3.

\section{Verification of the amplification efficiency and sensitivity of the developed PCR primers}

In order to assess the efficiency and sensitivity of the developed primers, qPCR assays were performed using tenfold serially diluted DNA (10-0.001 ng) of each species, and the individual statistical measurements were examined using a regression test. First, the quality of the extracted DNAs of each species was assessed by qPCR using universal plant primer (18S rRNA) pairs [27]. Then, the efficiencies of the designed primer pairs were examined. The results were in the range of $80-110 \%$, with strong correlation coefficient values $\left(R^{2}>0.99\right)$ for the target species (Table 4). However, no clear correlation coefficient was evident for the non-target species (e.g., see Fig. 3 for the O. japonica-specific primers, and Fig. S1 for the other species-specific primers). The slopes of the linear equations ranged from -3.12 to -3.89 . In order to confirm whether the PCR products were amplified from the target sequences, the amplicons were cloned and sequenced. As a result, all primers including the mismatched primers were verified to have amplified the target regions (Fig. 4). Generally, a discrimination analysis developed in a single laboratory would be confirmed for the easy implementation of a more reliable screening phase where necessary through inter-laboratory validation [28]. Therefore, we verified the performance of the developed $O$. japonica primers in two other laboratories. As a result, the developed $O$. japonica primers showed similar efficiencies of $80-110 \%$, with correlation coefficients of $R^{2}>0.99$ in both the laboratories (Fig. S2), suggesting that the developed markers would be acceptable for detecting the target species in commercial food products.

Table 4 Slope, correlation coefficient, efficiency, and Ct values obtained by qPCR assay using the developed primers

\begin{tabular}{|c|c|c|c|c|c|}
\hline Target species & Primer & Y (slope) & $R^{2}$ (correlation coefficients) & Efficiency $(\%)$ & $\mathrm{Ct}^{\mathrm{a}}$ \\
\hline \multirow[t]{3}{*}{ O. japonica } & $\operatorname{trn} T-L$ & -3.437 & 0.998 & 95.23 & 21.4 \\
\hline & $n d h F$ & -3.663 & 0.999 & 87.5 & 23.1 \\
\hline & rров & -3.348 & 0.999 & 98.9 & 22.8 \\
\hline \multirow[t]{8}{*}{ O. iwarenge and $O$. malacophyllus } & matK & $-3.652 /$ & $0.998 /$ & $84.26 /$ & 24 \\
\hline & & -3.642 & 0.999 & 88.88 & \\
\hline & $\operatorname{trnS}-G$ & $-3.489 /$ & $0.999 /$ & $93.46 /$ & 24.6 \\
\hline & & -3.525 & 0.999 & 92.18 & \\
\hline & $\operatorname{trn} Q-r p s 16$ & $-3.562 /$ & $0.999 /$ & $90.87 /$ & 23.3 \\
\hline & & -3.649 & 0.999 & 87.97 & \\
\hline & $y c f 2$ & $-3.395 /$ & $0.999 /$ & $97.04 /$ & 21.6 \\
\hline & & -3.497 & 0.999 & 93.17 & \\
\hline \multirow[t]{8}{*}{ O. latiellipticus and $O$. ramosus } & matK & $-3.61 /$ & $0.999 /$ & $89.2 /$ & 23.2 \\
\hline & & -3.648 & 0.999 & 87.98 & \\
\hline & $\operatorname{trn} T-L$ & $-3.385 /$ & $0.999 /$ & $97.41 /$ & 24 \\
\hline & & -3.286 & 0.999 & 101.54 & \\
\hline & $\operatorname{trn} Q-r p s 16$ & $-3.766 /$ & $0.996 /$ & $84.31 /$ & 26.7 \\
\hline & & -3.448 & 0.997 & 95.01 & \\
\hline & ITS & $-3.307 /$ & $0.998 /$ & $100.64 /$ & 23.2 \\
\hline & & -3.129 & 0.998 & 108.73 & \\
\hline O. margaritifolius & $n d h F$ & -3.899 & 0.998 & 80.49 & 24.4 \\
\hline
\end{tabular}

${ }^{\mathrm{a}} \mathrm{Ct}$ values represent the limit of detection of $10 \mathrm{pg}$ of DNA from each species using the species-specific primers 


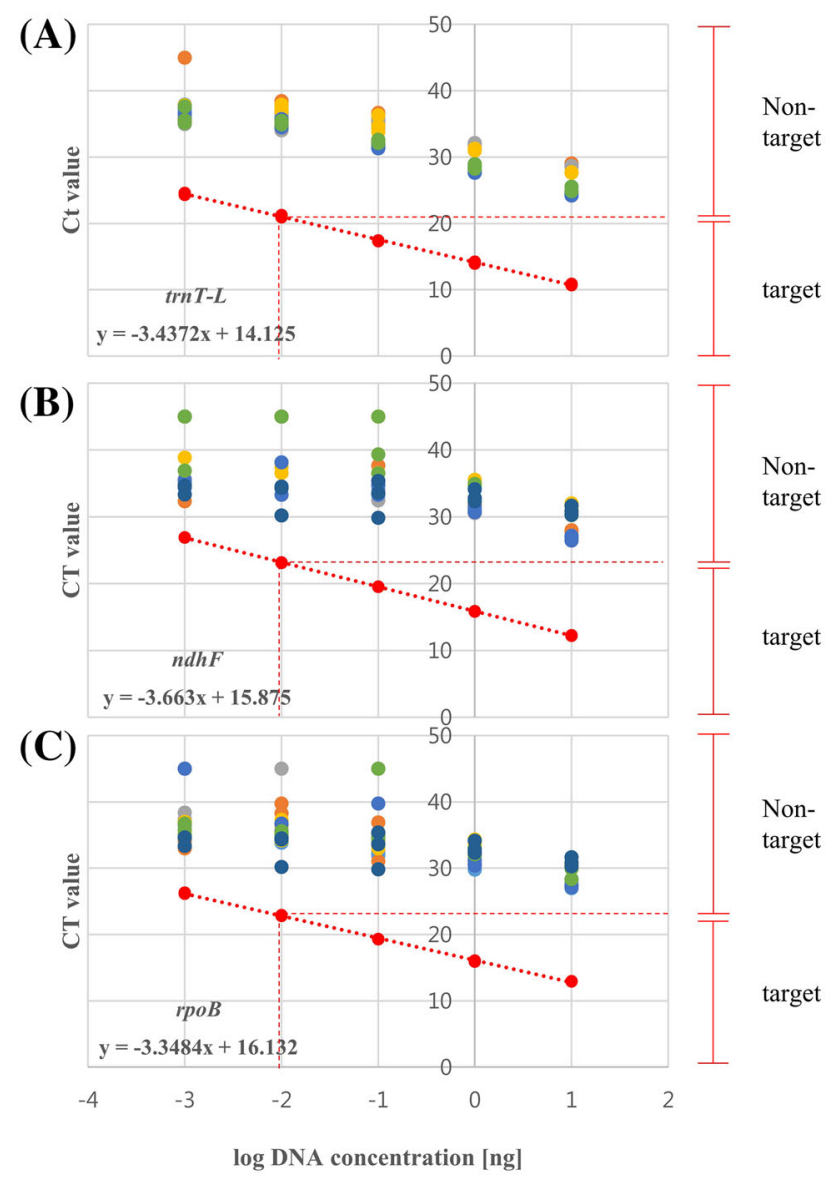

Fig. 3 Standard curves of $O$. japonica-specific primers obtained from tenfold serially diluted (from $10 \mathrm{ng}$ to $1 \mathrm{pg}$ ) DNA of $O$. japonica plants (target species). Non-target species means any other species. (A) O. japonica trnT-L primer; (B) O. japonica $n d h F$ primer; (C) $O$. japonica rpoB primer

\section{Verification of specificity of the developed $O$. japonica-specific primers}

In order to verify the specificity of the developed $O$. japonica-specific primers, which would be very useful for the discrimination of commercial $O$. japonica-based foods, binary mixtures of the DNAs from $O$. japonica and another Orostachys species were generated by tenfold serially mixed DNA (10-0.01\%) samples (Fig. 5) and subjected to qPCR. The primers were indeed found to be highly specific to $O$. japonica. For example, the $O$. japonica $r p o B$ primer could discriminate $0.01 \%$ of $O$. japonica DNA $(1 \mathrm{pg})$ from $99.99 \%$ of $O$. latiellipticus DNA, whereas it discriminated $0.1 \%$ O. japonica DNA from $99.9 \%$ of other species DNA, such as that of $O$. iwarenge and $O$. margaritifolius. The $O$. japonica trnT-L and $n d h F$ primers showed similar specificities for discrimination of the species (Fig. 5B). These results suggest that the primers could clearly discriminate at least $0.1 \%$ of $O$. japonica DNA in commercial food mixtures.

Subsequently, qPCR analysis was performed on artificially mixed $O$. japonica powder and wheat flour to confirm whether the $O$. japonica-specific primers could detect this species in the mixed products. All DNAs extracted from the various mixtures were quantified to $10 \mathrm{ng}$ for the qPCR analysis (Fig. 6). Consequently, the pure $O$. japonica powder $(100 \%)$ and the mixture of $O$. japonica $(90 \%)$ and wheat flour $(10 \%)$ were found to be amplified at the same $\mathrm{Ct}$ value with the three developed gene-based primers. With decreasing concentrations of $O$. japonica powder, the $\mathrm{Ct}$ values with the three developed primers increased
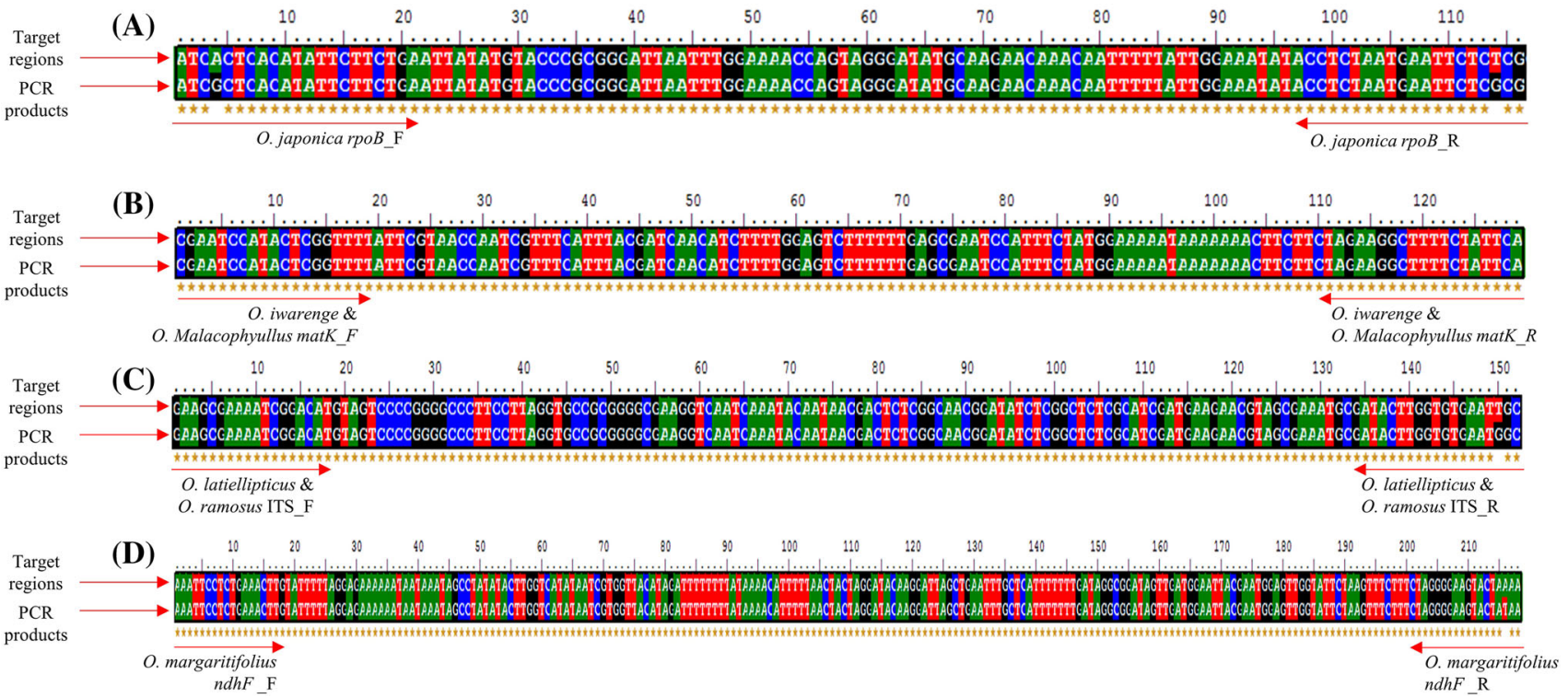

Fig. 4 Sequence comparison of the target regions and the PCR products obtained with the species-specific primers. (A) $O$. japonica rpoB primer; (B) O. iwarenge and O. malacophyllus matK primer; (C) O. latiellipticus and O. ramosus ITS primer; (D) O. margaritifolius $n d h F$ primer 
(A) O. japonica rpoB primer
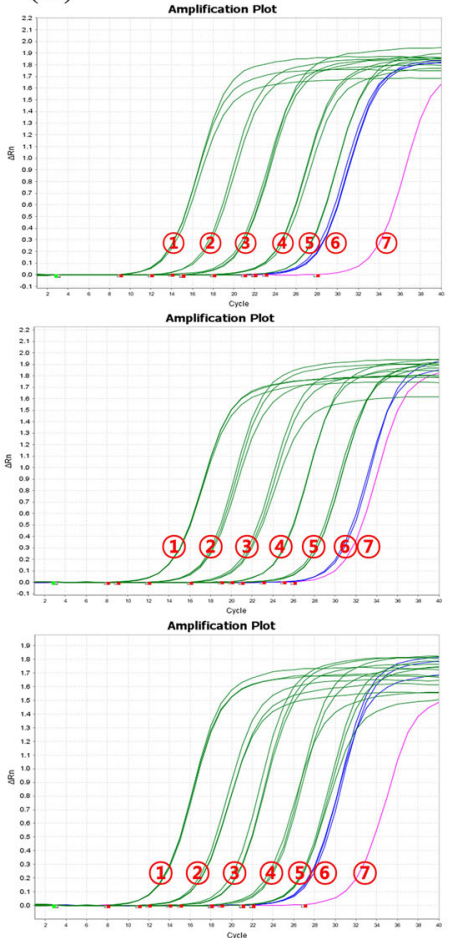

(B) O. japonica trnT-L primer

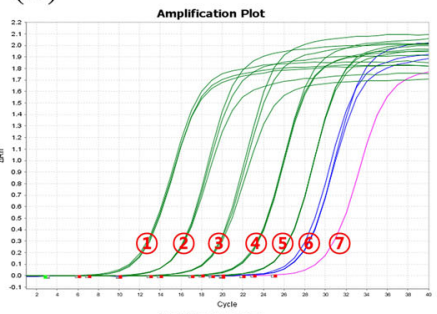

Amplification Plo
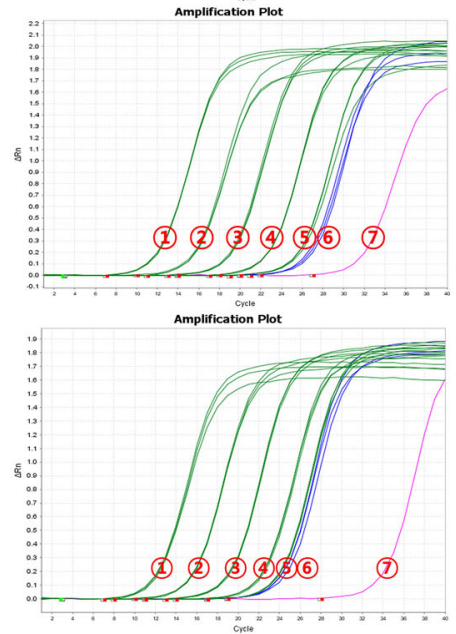

(C) O. japonica $n d h F$ primer

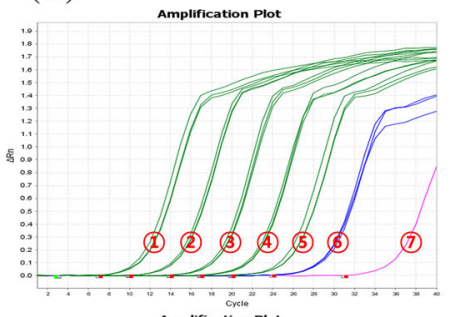

Amplification Plo
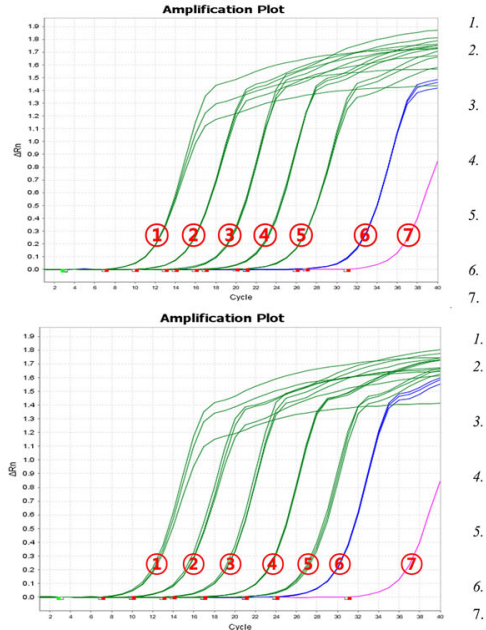

O. japonica $100 \%(10 \mathrm{ng})$

O. japonica $10 \%+$

O. iwarenge $90 \%$

O. japonica $1 \%+$

O. iwarenge $99 \%$

O. japonica $0.1 \%+$

O. iwarenge $99.9 \%$

O. japonica $0.01 \%+$

O. iwarenge $99.99 \%$

O. iwarenge $100 \%(10 \mathrm{ng})$

NTC (Negative Control)

O. japonica $100 \%$ (10ng)

O. japonica $10 \%+$

O. latiellipticus $90 \%$

O. japonica $1 \%+$

O. latiellipticus $99 \%$

O. japonica $0.1 \%+$

O. Iatiellipticus $99.9 \%$

O. japonica $0.01 \%+$

O. latiellipticus $99.99 \%$

O. latiellipticus $100 \%$ (10ng)

NTC (Negative Control)

O. japonica $100 \%$ (10ng)

O. japonica $10 \%+$

O. margaritifolius $90 \%$

O. japonica $1 \%+$

O. margaritifolius $99 \%$

O. japonica $0.1 \%+$

O. margaritifolius $99.9 \%$

O. japonica $0.01 \%+$

O. margaritifolius $99.99 \%$

O. margaritifolius $100 \%$ (10ng)

NTC (Negative Control)

Fig. 5 Real-time PCR assays of binary mixtures of DNA from the target species (O. japonica). (A) $O$. japonica rpoB primer; (B) $O$. japonica trnT-L primer; (C) O. japonica $n d h F$ primer

(A)

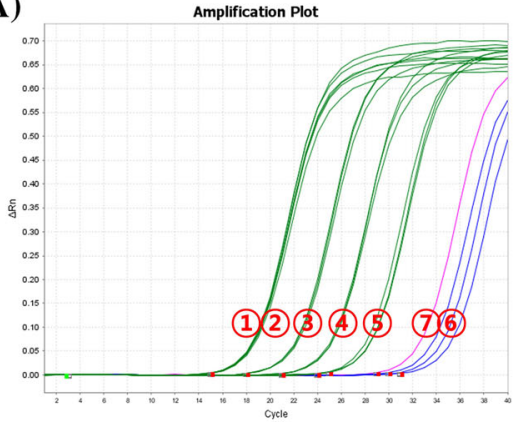

(B)

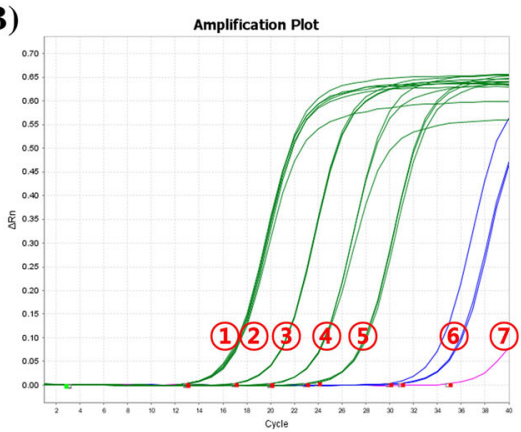

(C)

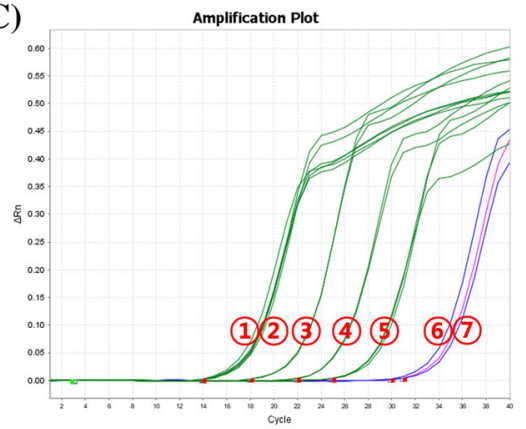

Fig. 6 Real-time PCR assays of artificial mixtures of $O$. japonica and wheat flour. 1. O. japonica powder $100 \% ; 2$. O. japonica powder (Haenam) $90 \%+$ wheat flour $10 \%$; 3. O. japonica powder $10 \%+$ wheat flour $90 \%$; 4. O. japonica powder $1 \%+$ wheat flour

gradually. Taking these results together, we concluded that the three $O$. japonica-specific primers were able to detect at least $0.1 \%$ of $O$. japonica powder additive in commercial food products.

\section{Application of the primers to commercial $O$. japonica food products}

The developed qPCR system was tested for its feasibility in detecting the Orostachys species in eight $O$. japonica food products, namely 3 powders, 4 dried samples, and 1 raw
99\%; 5. O. japonica powder $0.1 \%+$ wheat flour $99.9 \%$; 6 . wheat flour $100 \%$; 7. negative control (NTC). (A) O. japonica rpoB primer; (B) O. japonica trnT-L primer; (C) O. japonica $n d h F$ primer

sample, purchased from local markets. First, 18S rRNA primers were tested to verify whether the DNAs extracted from the food products were suitable for PCR amplification [5]. As a result, the 18S rRNA primers showed low $\mathrm{Ct}$ values (range 10.9-13.2) in all samples, confirming that the extracted DNA quality would be suitable for the qPCR assay. The developed primers were able to identify $O$. japonica DNA at concentrations of more than $10 \mathrm{pg}$, even though samples were admixed with the DNA of other species. Therefore, we concluded that $\mathrm{Ct}$ values for $10 \mathrm{pg}$ of DNA for all species-specific primers could be used as 


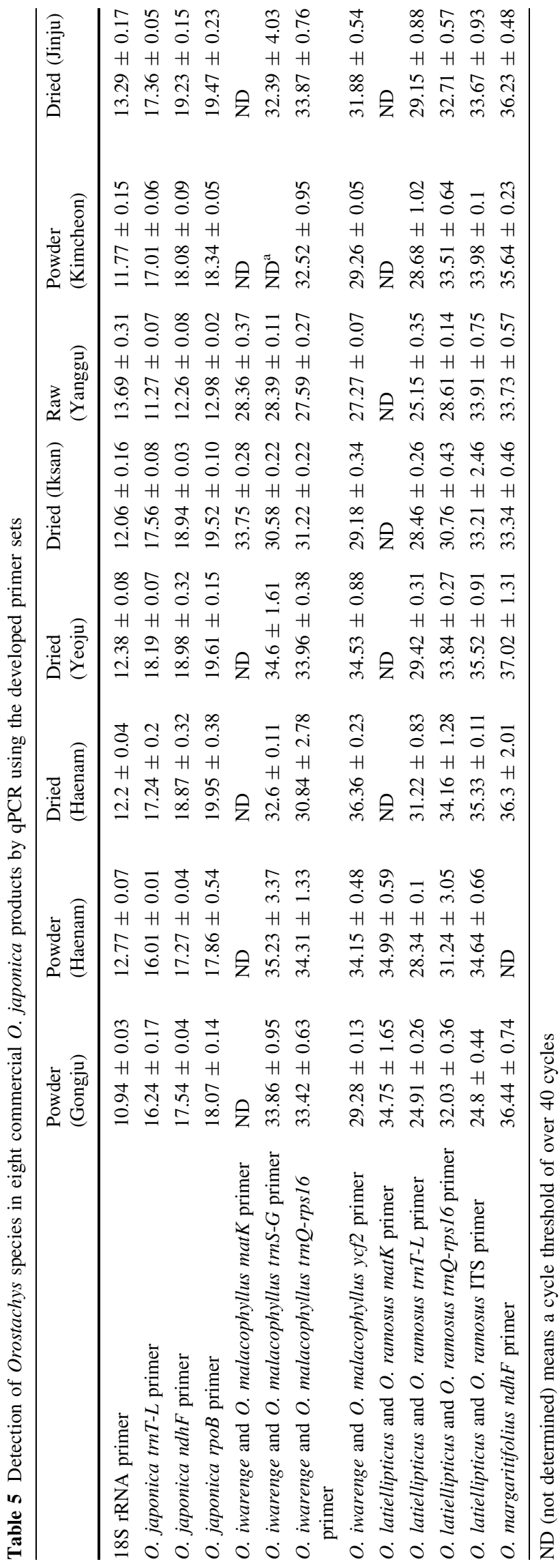

the LOD for the DNA of each species in commercial food products. The $\mathrm{Ct}$ values for $10 \mathrm{pg}$ of DNA for all our developed primers are provided in Table 4. qPCR results for the eight commercial $O$. japonica products using the developed primers are provided in Table 5. O. japonica DNA was detected in all eight commercial products, with low $\mathrm{Ct}$ values $(<20)$. However, none of the DNAs of the other Orostachys species were detected in all commercial products, because of high $\mathrm{Ct}$ values (over the detection limit of 10 pg DNA). Therefore, it suggests that the tested products did not contain any other Orostachys species except for $O$. japonica.

Recently, commercial $O$. japonica food products have gained attention owing to their various efficacies, such as antipyretic, hemostatic, diuretic, apoptotic cell death, and anticancer effects [7-9]. Several studies have been conducted to classify Orostachys species on the basis of numerous taxonomies [29], coenzyme electrophoresis [24], and DNA polymorphisms [30]. However, appropriate methodologies for the discrimination of $O$. japonica and/or other Orostachys species in commercial food products have not yet been developed.

Recently, the SYBR Green-based qPCR method has been used frequently as a useful tool for species-specific nucleotide detection and quantification. This method has several advantages, such as it is fast, reliable, easy to optimize compared with other biochemical methodologies, and also cheaper than other qPCR methods, such as the Taq-Man probe [31]. In fact, many researchers have developed SYBR Green-based qPCR techniques to detect other plant species in processed foods, such as hazelnut [32] and almond [33], DNA allergens [34], and C. wilfordii and $C$. auriculatum [5]. In this study, we have developed Orostachys primers for species-specific detection, tested their efficiency and sensitivity, and then applied them to commercial foods (Figs. 3, 5, 6, and S2). The developed primers and $\mathrm{qPCR}$ conditions were able to detect the presence of $O$. japonica or the absence of the other Orostachys species in commercial foods, suggesting that the methodology would be useful for verifying the authenticity of commercial $O$. japonica food products.

Acknowledgments This research was supported by a Grant (17162MFDS065) from Ministry of Food and Drug Safety in 2017.

\section{References}

1. Martirosyan DM, Singh J (2015) A new definition for functional food by FFC: creating functional food products using new definition. Introduction to functional food science, 3rd edn. Food Science Publisher, Ithaca 
2. Ozen AE, Pons A, Tur JA (2012) Worldwide consumption of functional foods: a systematic review. Nutr Rev 70(8):472-481

3. George VC, Dellaire G, Rupasinghe HV (2017) Plant flavonoids in cancer chemoprevention: role in genome stability. J Nutr Biochem 45:1-14

4. Johnson R (2014) Food fraud and "economically motivated adulteration" of food and food ingredients. Congressional Research Service, Washington

5. Kim JH, Moon J, Kang TS, Kwon K, Jang CS (2017) Development of cpDNA markers for discrimination between Cynanchum wilfordii and Cynanchum auriculatum and their application in commercial $C$. wilfordii food products. Applied. Biol Chem 60(1):79-86

6. Lee B, Kim H, Cho Y, Lee C (2001) Analysis of genetic relationship among korean native Orostachys species using RAPD. Korean J Hortic Sci Technol 19:159-162

7. Kim C, Park J, Lim J, Lee K, Chung G, Jeong H (2003) The activity of antioxidants and suppression of cancer cell proliferation in extracts of Orostachys japonicus A. berger. Korean J Med Crop Sci 11(1):31-39

8. Kwon J, Han K (2004) Effects of Orostachys japonicus A. berger on the immune system. Korean J Med Crop Sci 12(4):315-320

9. Ryu D, Baek G, Kim E, Kim K, Lee D (2010) Effects of polysaccharides derived from Orostachys japonicus on induction of cell cycle arrest and apoptotic cell death in human colon cancer cells. BMB Rep 43(11):750-755

10. Clegg MT, Gaut BS, Learn GH Jr, Morton BR (1994) Rates and patterns of chloroplast DNA evolution. Proc Natl Acad Sci USA 91(15):6795-6801

11. Shaw J, Lickey EB, Schilling EE, Small RL (2007) Comparison of whole chloroplast genome sequences to choose noncoding regions for phylogenetic studies in angiosperms: the tortoise and the hare III. Am J Bot 94(3):275-288

12. Selvaraj D, Sarma RK, Sathishkumar R (2008) Phylogenetic analysis of chloroplast matK gene from zingiberaceae for plant DNA barcoding. Bioinformation 3(1):24-27

13. Koch M, Haubold B, Mitchell-Olds T (2001) Molecular systematics of the brassicaceae: evidence from coding plastidic $m a t K$ and nuclear Chs sequences. Am J Bot 88(3):534-544

14. Steele KP, Vilgalys R (1994) Phylogenetic analyses of polemoniaceae using nucleotide sequences of the plastid gene matK. Syst Bot 126-142

15. Tamura MN, Yamashita J, Fuse S, Haraguchi M (2004) Molecular phylogeny of monocotyledons inferred from combined analysis of plastid $m a t K$ and $r b c L$ gene sequences. J Plant Res 117(2):109-120

16. Dong W, Liu J, Yu J, Wang L, Zhou S (2012) Highly variable chloroplast markers for evaluating plant phylogeny at low taxonomic levels and for DNA barcoding. PLoS ONE 7(4):e35071

17. Ford CS, Ayres KL, Toomey N, Haider N, Stahl JVA, Kelly LJ, Wikström N, Hollingsworth PM, Duff RJ, Hoot SB, Cowan RS, Chase MW, Wilkinson MJ (2009) Selection of candidate coding DNA barcoding regions for use on land plants. Bot J Linn Soc 159(1):1-11

18. Li X, Yang Y, Henry RJ, Rossetto M, Wang Y, Chen S (2015) Plant DNA barcoding: from gene to genome. Biol Rev 90(1):157-166
19. Álvarez I, Wendel JF (2003) Ribosomal ITS sequences and plant phylogenetic inference. Mol Phylogen Evol 29:417-434

20. Sanchiz Á, Ballesteros I, Martin A, Rueda J, Pedrosa M, Dieguez M, Rovira M, Cuadrado C, Linacero C (2017) Detection of pistachio allergen coding sequences in food products: a comparison of two real time PCR approaches. Food Control 75:262-270

21. Hwang S, Kim J, Moon J, Jang CS (2015) Chloroplast markers for detecting rice grain-derived food ingredients in commercial mixed-flour products. Genes Genom 37(12):1027-1034

22. Ramakers C, Ruijter JM, Deprez RHL, Moorman AF (2003) Assumption-free analysis of quantitative real-time polymerase chain reaction (PCR) data. Neurosci Lett 339(1):62-66

23. Ferreira T, Farah A, Oliveira TC, Lima IS, Vitório F, Oliveira EM (2016) Using real-time PCR as a tool for monitoring the authenticity of commercial coffees. Food Chem 199:433-438

24. Kim HD, Park KR (2005) Genetic variation in five species of Korean Orostachys (Crassulacea). Korean J Plant Taxon 35:295-311

25. Hayashi K, Hashimoto N, Daigen M, Ashikawa I (2004) Development of PCR-based SNP markers for rice blast resistance genes at the piz locus. Theor Appl Genet 108(7):1212-1220

26. Hirotsu N, Murakami N, Kashiwagi T, Ujiie K, Ishimaru K (2010) Protocol: a simple gel-free method for SNP genotyping using allele-specific primers in rice and other plant species. Plant Methods 6(1):12

27. Allmann M, Candrian U, Hffelein C, Lthy J (1993) Polymerase chain reaction (PCR): a possible alternative to immunochemical methods assuring safety and quality of food. Detection of wheat contamination in non-wheat food products. Z Lebensm Unters Forsch 196:248-251

28. Broeders S, Huber I, Grohmann L, Berben G, Taverniers I, Mazzara M, Roosens N, Morisset D (2014) Guidelines for validation of qualitative real-time PCR methods. Trends Food Sci Technol 37(2):115-126

29. Lee K, Kim H, Park K (2003) Numerical taxonomy of Korean Orostachys (crassulaceae). Korean J Plant Taxon 33(4):359-371

30. Nikulin AY, Nikulin VY, Gonctharova SB, Gontcharov AA (2015) ITS rDNA sequence comparisons resolve phylogenetic relationships in Orostachys subsection Appendiculatae (crassulaceae). Plant Syst Evol 301(5):1441-1453

31. Ponchel F, Toomes C, Bransfield K, Leong FT, Douglas SH, Field SL, Bell SM, Combaret V, Puisieux A, Mighell AJ (2003) Real-time PCR based on SYBR-green I fluorescence: an alternative to the TaqMan assay for a relative quantification of gene rearrangements, gene amplifications and micro gene deletions. BMC Biotechnol 3(1):18

32. Arlorio M, Cereti E, Coisson J, Travaglia F, Martelli A (2007) Detection of hazelnut (Corylus spp.) in processed foods using real-time PCR. Food Control 18(2):140-148

33. Pafundo S, Gulli M, Marmiroli N (2009) SYBR ${ }^{\circledR}$ GreenER $^{\mathrm{TM}}$ real-time PCR to detect almond in traces in processed food. Food Chem 116(3):811-815

34. Pafundo S, Gullì M, Marmiroli N (2010) Multiplex real-time PCR using SYBR ${ }^{\circledR}$ GreenER ${ }^{\mathrm{TM}}$ for the detection of DNA allergens in food. Anal Bioanal Chem 396(5):1831-1839 